\title{
Impact of Vitamin D Supplementation on Dry Eye in People with Low Serum Vitamin D
}

Saber Hamed El Said ${ }^{1}$, Ahmed Ibrahim Basiony ${ }^{1}$, Manar Mohammed Amin*1, Shaimaa Kamal El-deen²

Departments ${ }^{1}$ Ophthalmology and ${ }^{2}$ Internal Medicine, Faculty of Medicine, Menoufia University, Menoufia, Egypt *Corresponding author: Manar M. Amin, E-mail: manar.amin.sobih@gmail.com, Mobile (+20) 01558446656

\begin{abstract}
Background: Dry eye is a disease (DED) of the tear film and ocular surface, leading to ocular discomforts and pain. The pathophysiology of DED includes tear film instability and ocular surface inflammation. Vitamin D refers to a group of fat-soluble secosteroids responsible for enhancing intestinal absorption of calcium, iron, magnesium, phosphate, and zinc. It is not strictly a vitamin, and may be considered a hormone as its synthesis and activity occur in different locations.

Objective: This study aimed to investigate tear film function in patients with vitamin D deficiency.

Patient and methods: A case-control study included 75 eyes of 39 patients who were referred to the Ophthalmologic Clinic, Menoufia University during the period from March 2020 to December 2020. Patients of dry eye underwent tear break up time, Schirmer I test without anesthesia and special tests as well as measurement of serum vitamin D level were assessed.

Results: 25 -hydroxy vitamin D level of the studied group ranged from 6-18.50 ng/ml with mean value of $8.22 \pm 4.814$ $\mathrm{ng} / \mathrm{ml}$. Also, majority of studied sample (92.3\%) had bilateral affected eyes, followed by Gelinya (30\%). Schirmer's test, tear film breakup time (TFBUT) and ocular surface disease index (OSDI) were increased significantly when compared the values at baseline and after treatment to be after 10 weeks $(\mathrm{P}<0.001)$.

Conclusion: Vitamin D replacement appears to improve ocular surface health in patients with vitamin D deficiency. However, further clinical trials with a large sample size and control group are warranted to define the role of vitamin D. Vitamin D supplementation is an effective and useful treatment for patients with dry eye syndrome (DES) that is refractory to conventional treatment.
\end{abstract}

Keywords: Dry Eye, Schirmer I test, TFBUT, OSDI, Vitamin D.

\section{INTRODUCTION}

The tear film is a thin fluid layer providing a smooth surface over the cornea ${ }^{(\mathbf{1})}$. It is composed of 3 layers: the innermost mucin layer, an aqueous layer, and the outermost lipid layer ${ }^{(2)}$. Furthermore, the tear film is a complex mix of electrolytes, proteins (lipocalins, lactoferrin, transferrin, defensin, and lysozyme), phospholipids, oligopeptides, glycopeptides, and immunoglobulins. The tear film also includes a variety of surfactants, including surfactant proteins A and D. It is essential for the health of the eye that tears film components work in harmony ${ }^{(3)}$. Tears film stability is therefore, the result of the harmonious balance between tear components. A disturbance in one or more of the tear layers leads to the occurrence of dry eye disease (DED). DED is a disease of the tear film and ocular surface, leading to ocular discomforts and pain ${ }^{(4)}$. The pathophysiology of DED includes tear film instability and ocular surface inflammation ${ }^{(3)}$.

Vitamin D refers to a group of fat-soluble ketosteroids responsible for enhancing intestinal absorption of calcium, iron, magnesium, phosphate, and zinc. It is not strictly a vitamin, and may be considered a hormone as its synthesis and activity occur in different locations. Although commonly known for its role in calcium homeostasis, vitamin D also plays important roles in immune regulation, proliferation, differentiation, apoptosis, and angiogenesis. Vitamin D deficiency and genetic variations may cause a wide range of ocular pathologies, such as myopia, age-related macular degeneration, diabetic retinopathy, uveitis, and dry eye (5). Vitamin D deficiency may also cause dry eye, as studies have reported dry eye syndrome (DES) to be a localized autoimmune disease, and researchers recently hypothesized that vitamin $\mathrm{D}$ plays a role in the disorder because of its anti-inflammatory properties ${ }^{(6)}$. Dry eye, described by increased osmolarity of the tear film and inflammation of the ocular surface that cause ocular discomfort, visual disturbance, and tear film instability with potential damage to the ocular surface (7). Hyperosmolarity provides proinflammatory stress to the ocular surface ${ }^{(8)}$. The current study aimed to investigate tear film function in patients with vitamin $\mathrm{D}$ deficiency.

\section{PATIENTS AND METHODS}

A case-control study included 75 eyes of 39 patients who were referred to the Ophthalmologic Clinic, Menoufia University after exclusion of 4 cases who had sufficient 25 -hydroxy vitamin D. The study started from March 2020 to December 2020. 
Inclusion criteria: All patients were diagnosed with dry eye and low serum vitamin $D$ level.

Exclusion criteria: Patients excluded if they were presented with primary Sjogren syndrome, any other systemic rheumatic disease history,

vitamin B12 deficiency, history of smoking, current or recent drug use that could affect the lacrimal functional unit, active ocular infection or allergy, ocular surface scarring, previous eye surgery and current contact lens use.

All Patients were subjected to the following: Assessment of subjective symptoms of dry eye by using OSDI, Tear break up time (TFBUT), Schirmer I test without anesthesia and special tests as all patients with dry eye underwent measurement of serum vitamin D level to calculate the needed dose for supplementation.

Examination included: General examination including (Skin (e.g., scleroderma, facial changes consistent with rosacea, seborrhea) and Cranial nerves functions [e.g., cranial nerve V (trigeminal), cranial nerve VII (facial)].

Ocular examination included: External eye examination including Eye lid skin for rosacea, seborrhea, tear glands, swelling in the lateral upper lid area, eyelids ectropion, entropion, eversiopuncti lacrimalis, lid defect, scars, redness/swelling of lid margin, eyelid closure, voluntary, involuntary, Bell's phenomenon, proptosis, [Blinking, frequency, increased, (normal value approx. 12 blink per minute)] and Meibomian glands (sufficient in number and gland openings. Blocked G-Corneal sensation was assessed when trigeminal nerve dysfunction was suspected (history of HZO).

Slit-lamp Examination included: Examination for tear film abnormalities including Tear meniscus height: In dry eye it is concave, decreased in volume and may be absent in severe cases (normal tear meniscus height $=268 \pm 68 \mu \mathrm{m}$ ), tear meniscus floater: They could be seen as tiny bits of debris in the upper and lower tear menisci and mucous strands.

Conjunctival Surface examination including dilated vessels, strings of mucus, loss of luster and elasticity of the conjunctiva. Papillary conjunctivitis, which was a non-specific sign results from irritation (Nodular, sectorial, diffuse episcleritis and scleromalacia provided evidence for associated connective tissue disease). Sub-epithelia fibrosis and keratinzation of the conjunctiva, we inspected carefully the inferior and superior fornices, looking for symblepharon in cases of cicatrizing diseases, the lid parallel conjunctival folds (LIPCOFs) (conjunctivochaisis) and Bitot's spot. Corneal examination including punctate epitheliopathy, corneal filaments( short tails usually less than $2 \mathrm{~mm}$ filaments), mucous plaques(semi- transparent, white to grey slightly elevated lesion of different shapes and sizes) and corneal thinning and perforation (it can complicate Kerato-conjunctivitis sicca especially in the presence of rheumatoid arthritis).

Anterior chamber examination: In patients with connective tissue disease for anterior uveitis detection. Fundus examination as a routine examination in hypertensive and diabetic patients and in patients with connective tissue disease for posterior uveitis detection and by slit-lamp biomicroscopy. Diagnostic Tests for tear Quality: Tear breakup time test (fluorescein is applied into the lower fornix and the patient is first asked to blink several times and then to avoid blinking. A broad slit-lamp beam with cobalt blue filter is used to scan the tear film. The presence of black spots or lines indicates the appearance of dry spots in the tear film. Tear film breakup time (TBUT) is the interval between the blink and the appearance of the first randomly distributed dry spot. A TBUT of less than 10 seconds is considered abnormal.

Schirmer test indication including Evaluation of dry eye (Measures aqueous tear production). Schirmer 1 (without anesthetic): it measure's baseline and reflex secretion and evaluate function of main lacrimal gland, whose secretory activity is stimulated by the irritating nature of filter paper. Materials required: 5 X $35 \mathrm{~mm}$ of Schirmer's strip or Whatman filter paper.

\section{Ethical consent:}

An approval of the study was obtained from Menoufia University Academic and Ethical Committee. Every patient signed an informed written consent for acceptance of the operation.

\section{Statistical Analysis}

Results were tabulated and statistically analyzed using standard computer program using MICROSOFT EXCEL 2017 and SPSS V.25 program for MICROSOFT WINDOWS 10. Two types of statistics were done: Descriptive statistics that includes the description of data was in the form of mean \pm SD for quantitative data, and frequency and proportion for qualitative data. Paired $t$ test and chi-square test were used. $\mathrm{P}$ value $\leq 0.05$ considered a significant level.

\section{RESULTS}

In the current study, age of the studied patients ranged from 20-67 years with a mean value of 51.60 \pm 11.921 years. Male cases were $18(41.9 \%)$ while female cases were $25(58.1 \%)$ as shown in table (1).

In the current study, 25-hydroxy vitamin D level of the studied group ranged from 6-18.50 ng/ml with a mean value of $8.22 \pm 4.814 \mathrm{ng} / \mathrm{ml}$. Also, majority of studied sample $(92.3 \%)$ had bilateral affected eyes, followed by Gelinya (30\%), as shown in table (2). 
Table (1): Distribution of studied sample according to patient's demographic data.

\begin{tabular}{|l|c|c|}
\hline \multicolumn{1}{|c|}{ Variables } & Number & Percent \\
\hline Age (years) & \multicolumn{2}{|c|}{} \\
$<55$ & 22 & 51.2 \\
$\geq 55$ & 21 & 48.8 \\
\hline Mean \pm SD & \multicolumn{2}{|c|}{$51.60 \pm 11.921$} \\
\hline Sex & \multicolumn{2}{|c|}{} \\
Male & 18 & 41.9 \\
Female & 25 & 58.1 \\
\hline
\end{tabular}

Table (2): Distribution of studied sample according to patient's 25-hydroxy vitamin D and affected eye side in patients with vitamin D deficiency.

\begin{tabular}{|l|c|c|}
\hline \multicolumn{1}{|c|}{ Variables } & No. & \% \\
\hline 25-hydroxy vitamin D/ (ng/ml) & \multicolumn{2}{|c|}{$8.22 \pm 3.814$} \\
Mean \pm SD & $\mathbf{4}$ & $\mathbf{9 . 3}$ \\
\hline Normal level of Vitamin D & $\mathbf{3 9}$ & $\mathbf{9 0 . 7}$ \\
\hline Vitamin D Deficiency & & \\
\hline Side & 2 & 5.1 \\
Left & 1 & 2.6 \\
Right & 36 & 92.3 \\
Bilateral & 39 & 100.0 \\
Total & & \\
\hline
\end{tabular}

In the present study, Schirmer 1 ranged from 1.0-12.5 with a mean value of $6.48 \pm 3.006$. Also, TFBUT ranged from $0-8 \mathrm{sec}$ with a mean value of $3.62 \pm 2.104 \mathrm{sec}$. OSDI ranged from 13-48 with a mean value of $32.58 \pm 9.607$. About $17.9 \%, 28.3 \%$ and $53.8 \%$ of the studied group were mild, moderate and severe OSDI grades at one day before treatment, respectively as shown in table (3).

Table (3): Distribution of studied sample according to patient's Schirmer 1, TFBUT, ODSI and ODSI grades at one day before treatment in patients with vitamin D deficiency

\begin{tabular}{|l|c|c|}
\hline \multicolumn{1}{|c|}{ Variables } & Min. - Max. & Mean \pm SD \\
\hline Schirmer's test (n=75 eye) & $1.0-12.5$ & $6.48 \pm 3.006$ \\
\hline TFBUT (sec) (n=75 eye) & $0-8$ & $3.62 \pm 2.104$ \\
\hline OSDI (n=39) & $13-48$ & $32.58 \pm 9.607$ \\
\hline OSDI grades & No & \% \\
Mild & 7 & 17.9 \\
Moderate & 11 & 28.3 \\
Severe & 21 & 53.8 \\
Total & 39 & 100.0 \\
\hline
\end{tabular}

TFBUT: Tear Film Breakup time

OSDI: Ocular Surface Disease Index.

Regarding Schirmer's test, TFBUT and OSDI grades were increased significantly when comparing the values at baseline with after treatment with $(\mathrm{P}<0.001)$. While, OSDI were decreased significantly when comparing the values at baseline with after treatment to be after 10 weeks $16.42 \pm 9.254(\mathrm{P}<0.001)$ as shown in table $(4)$. 
Table (4): Comparison between baselines and after treatment according to Schirmer's TFBUT, OSDI and OSDI grades in patients with vitamin D deficiency

\begin{tabular}{|c|c|c|c|c|c|c|c|c|}
\hline & \multirow{2}{*}{\multicolumn{2}{|c|}{ Baseline }} & \multicolumn{6}{|c|}{ After Treatment } \\
\hline & & & \multicolumn{2}{|c|}{2 weeks } & \multicolumn{2}{|c|}{6 weeks } & \multicolumn{2}{|c|}{10 weeks } \\
\hline \multicolumn{9}{|c|}{ Schirmer's test } \\
\hline Min.-Max. & \multicolumn{2}{|c|}{$1-12.5$} & \multicolumn{2}{|c|}{$3-14$} & \multicolumn{2}{|c|}{$2-13.5$} & \multicolumn{2}{|c|}{$1.5-13$} \\
\hline Mean \pm SD & \multicolumn{2}{|c|}{$6.48 \pm 3.006$} & \multirow{2}{*}{\multicolumn{2}{|c|}{$\begin{array}{c}8.02 \pm 2.856 \\
<0.001 *\end{array}$}} & \multicolumn{2}{|c|}{$7.45 \pm 2.859$} & \multicolumn{2}{|c|}{$7.14 \pm 2.567$} \\
\hline $\mathrm{P}$ value & \multicolumn{2}{|c|}{----- } & & & \multicolumn{2}{|c|}{$<0.001^{*}$} & \multicolumn{2}{|c|}{$<0.001 *$} \\
\hline \multicolumn{9}{|l|}{ BUT } \\
\hline Min.-Max. & \multicolumn{2}{|c|}{$0-8$} & \multicolumn{2}{|c|}{$2-10$} & \multicolumn{2}{|c|}{$1-12$} & \multicolumn{2}{|c|}{$1-10$} \\
\hline Mean \pm SD & \multicolumn{2}{|c|}{$3.62 \pm 2.104$} & \multicolumn{2}{|c|}{$5.27 \pm 1.862$} & \multicolumn{2}{|c|}{$4.51 \pm 2.194$} & \multicolumn{2}{|c|}{$4.83 \pm 2.098$} \\
\hline P value & \multicolumn{2}{|c|}{----- } & \multicolumn{2}{|c|}{$<0.001^{*}$} & \multicolumn{2}{|c|}{$<0.001 *$} & \multicolumn{2}{|c|}{$<0.001 *$} \\
\hline \multicolumn{9}{|l|}{ OSDI } \\
\hline Min. -Max. & \multicolumn{2}{|c|}{$13-48$} & \multicolumn{2}{|c|}{$8-36$} & \multicolumn{2}{|c|}{$7-36$} & & \\
\hline Mean \pm SD & 32.5 & .607 & & 101 & 23. & .595 & 16.4 & 254 \\
\hline $\mathrm{P}$ value & & & & & & & & \\
\hline OSDI grades & & & & & & & & \\
\hline & NO. & $\%$ & NO. & $\%$ & NO. & $\%$ & NO. & $\%$ \\
\hline Normal & 0 & 0 & 7 & 17.9 & 6 & 15.4 & 15 & 38.5 \\
\hline Mild & 7 & 17.9 & 13 & 33.3 & 12 & 30.7 & 18 & 46.2 \\
\hline Moderate & 11 & 28.3 & 17 & 43.7 & 15 & 38.5 & 5 & 12.7 \\
\hline Severe & 21 & 53.8 & 2 & 5.1 & 6 & 15.4 & 1 & 2.6 \\
\hline $\mathrm{P}$ value & & & & & & & & \\
\hline
\end{tabular}

TFBUT: Tear Film Breakup time

OSDI: Ocular Surface Disease Index

* significantly

\section{DISCUSSION}

Dry eye disease is a multifactorial disease of the ocular surface characterized by a loss of homeostasis of the tear film and is accompanied by ocular symptoms, in which tear film instability and hyperosmolarity, ocular surface inflammation and damage, and neurosensory abnormalities play etiological roles ${ }^{(9)}$. The anti-inflammatory effect of activated vitamin $\mathrm{D}$ is achieved by blocking the activation of T-helper cells and cytotoxic T cells, and reducing the production of inflammatory mediators such as interleukin (IL)-2, IL-6, IL-8, and IL-12. Furthermore, vitamin D inhibits inflammatory factors such as C-reactive protein (CRP), tumor necrosis factor (TNF)- $\alpha$, IL-1, and IL-6, and induces IL-10 production ${ }^{(10)}$. Thus, the aim of this study was to evaluate the role of vitamin D supplementation on dry eye in people with low serum vitamin D. This was a prospective study, which was conducted on 43 patients with dry eye who are referred to the Ophthalmologic Clinic of Menoufia University.

Analysis of our findings as regard, sociodemographic characteristics of the participant patients revealed that age ranged from 20-67 years with a mean value of $51.60 \pm 11.921$ years. Male cases were $18(41.9 \%)$ while female cases were $25(58.1 \%)$. These are in agreement with findings, of Bae et al. ${ }^{(11)}$ which concluded that the mean age of the patients was $58.21 \pm 12.94$ years and included 21 men and 84 women. In another prospective-randomized study of Watts et al. ${ }^{(12)}$ in which 90 patients with DED with deficient serum $25(\mathrm{OH}) \mathrm{D}$ levels were randomized into three groups and were given treatment for dry eye. Out of 90 patients, 52 were females and 38 were males with a mean age of $44.87 \pm 14.77$ (range $=20-80$ ) years. Among the many etiological factors, an association of Vitamin D deficiency with DES and tear film insufficiency has received attention in recent studies. The prevalence of vitamin D deficiency in India has been reported to be $80 \%-90 \%$ in most studies ${ }^{(13)}$, a study of Watts et al. ${ }^{(12)}$ and Aparna et al. ${ }^{(14)}$ defined the deficiency as a serum vitamin D level of $<20$ $\mathrm{ng} / \mathrm{ml}$. However, another study of Yoon et al. ${ }^{(15)}$ has taken $<12 \mathrm{ng} / \mathrm{ml}$ as the cutoff.

In the current study, we found that 25-hydroxy vitamin $\mathrm{D}$ of the studied group ranged from 1.70-18.50 $\mathrm{ng} / \mathrm{ml}$ with a mean value of $5.22 \pm 4.814 \mathrm{ng} / \mathrm{ml}$. Our findings are compared by the study of Shahraki $\boldsymbol{e t}$ al. (16) in which the mean vitamin D level in patients with dry eye syndrome was $20.86 \pm 10.88 \mathrm{ng} / \mathrm{dl}$. Many studies of demonstrated the relationship between dry eye syndrome and vitamin D deficiency. Yildirim $\boldsymbol{e t}$ al. ${ }^{(17)}$ demonstrated significant correlation between vitamin D deficiency and TFBUT, Schirmer II and OSDI. Therefore, they concluded that vitamin D deficiency may display a protective role in the development of dry eye, possibly by improving dry eye parameters and decreasing ocular surface inflammation. Demirci et al. ${ }^{(18)}$ demonstrated that vitamin D deficiency is associated with tear hyperosmolarity and tear film dysfunction. On the other hand, other studies didn't find significant 
relationship between dry eye and vitamin D deficiency (15-17).

In the current study, Schirmer 1 ranged from $1.0-12.5$ with a mean value $6.48 \pm 3.006$. TFBUT ranged from $0-8 \mathrm{sec}$ with a mean value of $3.62 \pm 2.104$ sec. OSDI ranged from 13-48 with a mean value of $32.58 \pm 9.607$. In another study done by Elagamy and Bawazir (19), there were no statistically significant differences regarding Schirmer II test values, TFBUT measurements, and OSDI scores between the two groups $(\mathrm{P}>0.05)$. The mean of TFBUT was $6.9 \pm 3.7$ $\mathrm{sec}$ in group 1 and $6.7 \pm 4.1 \mathrm{sec}$ in group 2 . The mean of Schirmer II test values was $23.1 \pm 7.7 \mathrm{~mm}$ in group 1 and $24.3 \pm 7.0 \mathrm{~mm}$ in group 2. The mean of OSDI scores was $19.2 \pm 18.4$ in group 1 and $15.0 \pm 19.5$ in group 2 .

In the present study, after treatment Schirmer's test increased significantly when comparing the values at baseline and after treatment to be after 10 weeks with a mean value of $7.14 \pm 2.567$. TFBUT increased significantly when comparing the values at baseline and after treatment to be after 10 weeks with a mean value of $4.83 \pm 2.098$, while, OSDI decreased significantly when comparing the values at baseline and after treatment to be after 10 weeks with a mean value of $16.42 \pm 9.254$. OSDI grades showed highly statistically significant improvement when comparing the results at baseline with results after treatment. In comparison with the study of Bae et al. (11) in which tear secretion by Schirmer test was $6.69 \pm 3.92 \mathrm{~mm}$ at pre-treatment, $8.64 \pm 6.32 \mathrm{~mm}$ after 2 weeks, $8.92 \pm$ $7.60 \mathrm{~mm}$ after 6 weeks, and $8.40 \pm 7.16 \mathrm{~mm}$ after 10 weeks. OSDI was $34.33 \pm 24.88$ at pre-treatment, $29.25 \pm 23.35$ after 2 weeks, and $21.07 \pm 16.52$ after 10 weeks. TBUT was $3.16 \pm 2.27 \mathrm{sec}$ at pre-treatment, increased to $5.58 \pm 2.44 \mathrm{sec}$ after 2 weeks and to 5.19 $\pm 2.34 \mathrm{sec}$ after 6 weeks, before returning to the pretreatment levels after 10 weeks. In another recent study done by Karaca et al. ${ }^{(20)}$, which aimed to analyze the clinical outcomes of the ocular surface in patients with vitamin $\mathrm{D}$ deficiency after oral replacement, and reported that Schirmer I test score increased from $13.10 \pm 8.01 \mathrm{~mm}$ at baseline to $17.33 \pm 7.29 \mathrm{~mm}$ after 24 weeks. TBUT improved from $5.53 \pm 3.12 \mathrm{sec}$ to $9.13 \pm 3.01 \mathrm{sec}$. Tear osmolarity was $307.4 \pm 15.4$ $\mathrm{mOsm} / \mathrm{L}$ at baseline and $302.7 \pm 10.6$ after 24 weeks. The OSDI score improved throughout the entire study period. On the other hand, in the current study, we found that the increase in Schirmer's test showed no statistically significant differences between patients in the age $<55$ years and patients with age $\geq 55$ years when comparing the values at baseline with values after treatment but the increase in result was higher in patients in the age $<55$ years than in patients with age $\geq 55$ years. Moreover, the increasing in the BUT showed no statistically significant differences between patients in the age $<55$ years and patients with age $\geq$
55 years when comparing the values at baseline with values after treatment but the increase in result was higher in patients in the age $<55$ years than in patients with age $\geq 55$ years. Furthermore, the decrease in the OSDI showed no statistically significant differences between patients in the age $<55$ years and patients with age $\geq 55$ years when comparing the values at baseline with values after treatment but the decrease in result was greater in patients in the age $<55$ years than in patients with age $\geq 55$ years.

The effects of vitamin D supplementation on DES were analyzed according to gender, in the current study, the increase in Schirmer's test showed no statistically significant differences between male patients and female patients when comparing the values at baseline with values after treatment but the increase in result was higher in male patients than in female patients.

The present study revealed that the increase in BUT showed no statistically significant differences between male patients and female patients when comparing the values at baseline with values after treatment but the increase in result was higher in male patients than in female patients. Our results are supported by the study of Bae et al. (11), which demonstrated that the effects of vitamin D supplementation on DES were analyzed according to gender. The TBUT in males was increased after 2 weeks compared to pre-treatment and in female it was increased after 2 and 6 weeks compared to pretreatment.

In the study on our hands, the decrease in the OSDI showed no statistically significant differences between male patients and female patients when comparing the values at baseline with values after treatment but the decrease in result was greater in male patients than in female patients. Similar to our findings, Bae et al. ${ }^{(11)}$ reported that OSDI score in men was lower at 6 weeks compared to pre-treatment and in women it was lower at 10 weeks compared to pretreatment.

In a study of Elagamy and Bawazir (19), concerning assessment of dry eye parameters according to the gender in group 1 with vitamin D deficiency, the mean of Schirmer II test values (mm) was significantly lower $(21.3 \pm 7.5)$ in females compared to $(25.5 \pm 7.3)$ in males. While no statistically significant differences regarding superficial punctate staining. The mean of TBUT (sec) was lower $(6.9 \pm 3.5)$ in males compared to $(7.0 \pm 4.0)$ in females. The mean of OSDI scores was lower (18.3 $\pm 17.5)$ in males compared to $(19.9 \pm 19.2)$ in females. The effect of vitamin $\mathrm{D}$ has been reported to differ according to gender in Dawson-Hughes et al. ${ }^{(21)}$ and Halfon et al. (22). Vitamin D is an important factor in estrogen biosynthesis and estrogen signaling, and has anti-estrogenic activity ${ }^{(23)}$. 


\section{CONCLUSION}

Vitamin D replacement appears to improve ocular surface health in patients with vitamin D deficiency. However, further clinical trials with a large sample size and control group are warranted to define the role of vitamin D. Vitamin D supplementation promoted tear secretion, reduced tear instability, and reduced inflammation at the ocular surface and eyelid margin. Furthermore, vitamin D supplementation improved the symptoms of DES. In addition, vitamin D supplementation is an effective and useful treatment for patients with DES that is refractory to conventional treatment.

\section{REFERENCES}

1. Gipson I (2007): The ocular surface: the challenge to enable and protect vision: the Frieden Wald lecture. Investigative Ophthalmology and Visual Science, 48 (10): 4391-8.

2. Holly F, Lemp M (1977): Tear physiology and dry eyes. Survey of Ophthalmology, 22 (2): 69-87.

3. Dartt D, Willcox M (2013): Complexity of the tear film: importance in homeostasis and dysfunction during disease. Experimental Eye Research, 117: 1-6.

4. Brauer L, Kindler C, Jager K et al. (2007): Detection of surfactant proteins A and D in human tear fluid and the human lacrimal system. Investigative Ophthalmology \& Visual Science, 48 (9): 3945-53.

5. Autier P, Boniol M, Pizot C et al. (2014): Vitamin D status and ill health: a systematic review. The Lancet Diabetes \& Endocrinology, 2 (1): 76-89.

6. Stevenson W, Chauhan S, Dana R (2012): Dry eye disease: an immune-mediated ocular surface disorder. Archives of Ophthalmology, 130 (1): 90-100.

7. Lemp M, Foulks G (2007): The definition and classification of dry eye disease. Ocul Surf., 5 (2): 7592.

8. Luo L, Li D, Corrales R et al. (2005): Hyperosmolar saline is a proinflammatory stress on the mouse ocular surface. Eye \& Contact Lens, 31 (5): 186-93.

9. Aparna P, Muthathal S, Nongkynrih B et al. (2018): Vitamin D deficiency in India. Journal of Family Medicine and Primary Care, 7 (2): 324-329.

10. Craig J, Nichols K, Akpek E et al. (2017): TFOS DEWS II Definition and Classification Report. Ocul Surf., 15 (3): 276-283.

11. Bae S, Shin Y, Kim H et al. (2016): Vitamin D supplementation for patients with dry eye syndrome refractory to conventional treatment. Scientific Reports, 6 (1): 1-0.

12. Watts $P$, Sahai A, Kumar $P$ et al. (2020): A prospective study to assess the role of vitamin D individually and in combination with cyclosporine in the treatment of dry eye in patients with deficient serum $25(\mathrm{OH}) \mathrm{D}$ levels. Indian Journal of Ophthalmology, 68 (6): 1020-25.

13. Rosenthal P, Borsook D (2016): Ocular neuropathic pain. British Journal of Ophthalmology, 100 (1): 128 34.

14. Aparna $P$, Muthathal $S$, Nongkynrih B et al. (2018): Vitamin D deficiency in India. Journal of Family Medicine and Primary Care, 7 (2): 324-29.

15. Yoon S, Bae S, Shin Y et al. (2016): Low serum 25hydroxyvitamin $\mathrm{D}$ levels are associated with dry eye syndrome. PLoS One, 11 (1): 147847.

16. Shahraki K, Farzaneh M, Ghasemi Boroumand $P$ et al. (2018): Serum Vitamin D Level in Patients with Dry Eye Syndrome. Bina Journal of Ophthalmology, 24 (1): 9-15.

17. Yildirim P, Garip Y, Karci A et al. (2016): Dry eye in vitamin $\mathrm{D}$ deficiency: more than an incidental association. International Journal of Rheumatic Diseases, 1s: 49-54.

18. Demirci G, Erdur S, Ozsutcu M et al. (2018): Dry eye assessment in patients with vitamin D deficiency. Eye \& Contact Lens, 44: 62-65.

19. Elagamy A, Bawazir S (2019): Dry Eye Evaluation in Saudi Patients with Vitamin D Deficiency. J Ophthalmol Eye Care, 2 (1): 105-109.

20. Karaca E, Kemer Ö, Özek D et al. (2020): Clinical outcomes of ocular surface in patients treated with vitamin D oral replacement. Arquivos Brasileiros de Oftalmologia, 4: 312-7.

21. Dawson-Hughes B, Harris S, Krall E et al. (1997): Effect of calcium and vitamin D supplementation on bone density in men and women 65 years of age or older. New England Journal of Medicine, 337 (10): 670-6.

22. Halfon M, Phan O, Teta D (2015): Vitamin D: a review on its effects on muscle strength, the risk of fall, and frailty. Bio Med Research International, 2015:953241.

23. Kinuta $K$, Tanaka $H$, Moriwake $T$ et al. (2000): Vitamin D is an important factor in estrogen biosynthesis of both female and male gonads. Endocrinology, 141 (4): 1317-24. 